Igor Ille*, Sebastian Mojrzisch and Jens Twiefel

\title{
Process Emulation System for High-Power Piezoelectric Ultrasonic Actuators
}

\begin{abstract}
Ultrasonic actuators are used for a wide field of applications. The vibration energy can be used to realize many processes like ultrasonic welding or bonding. Furthermore there are many processes which run more efficient and faster combined with ultrasonic vibration like ultrasonic-assisted turning or drilling. Piezoelectric transducers are the main part of those applications. Most of the applications have a time-variant load behavior and need an amplitude feedback control to guarantee a stable process. To ensure correct function tests of the feedback control systems have to be done. In this case the processes have to be executed in association with a high number of cycles. To emulate the behavior of the environment the automotive and aerospace industries use hardware in the loop systems since a long time but there is no such a method for ultrasonic systems. This paper presents a method to realize high dynamic load emulation for different ultrasonic applications. Using a piezoelectric transformer it is possible to reproduce load curves by active damping on the secondary side of the transformer using a current proportional digital feedback circuit. A theoretical and experimental study of hardware in the loop system for ultrasonic applications is given by this paper. The present system allows testing a wide field of feedback control algorithms with high flexibility and a high number of cycles by utilization of low-cost components. This proceeding decreases design periods in association with feedback control.
\end{abstract}

Keywords: ultrasonic, piezoelectric transformer, load emulation, process emulation, hardware in the loop

DOI 10.1515/ehs-2014-0036

\footnotetext{
*Corresponding author: Igor Ille, Institute of Dynamics and Vibration Research, Gottfried Wilhelm Leibniz University of Hannover, Hannover, Germany, E-mail: ille@ids.uni-hannover.de Sebastian Mojrzisch, Jens Twiefel, Institute of Dynamics and Vibration Research, Gottfried Wilhelm Leibniz University of Hannover, Hannover, Germany
}

\section{Introduction}

Piezoelectric transducers are driven in their resonance due to energetic reasons. Most of the processes realized or assisted by ultrasonic vibration have time-variant load behavior like Ahmed et al. (2007) show using the example of ultrasonic assisted turning. The time-variant load behavior results in a dynamic resonance frequency shift and an influence on the vibration amplitude. With rising load the vibration amplitude decreases. To ensure a stable amplitude and operation point a feedback control has to be used. The feedback control algorithms can be tested using the real system or by realizing an approach to emulate the process of interest. In case of emulation the time-variant load behavior can be identified using measured current and voltage at the terminals of the transducer. How to model a piezoelectric transducer including a load can be derived from Littmann (2001).

The process emulation discussed in this paper is realized using a piezoelectric transformer. The transformer consists of two against each other bolted piezoelectric transducers. One of the transducers is driven in resonance. The second transducer is used for time specific damping. The design criteria and model for a piezoelectric transformer are explained in detail by Hemsel (2006) and Littmann (2004). The feedback control used in this contribution is described by Twiefel et al. (2008).

\section{Model Description}

\section{Piezoelectric Transducer}

A piezoelectric transducer can be modeled in electrical equivalent form as shown in Figure 1. The circuit consists of an oscillating circuit and a parallel capacitance. The load resistance $R_{\mathrm{L}}$ and the load capacitance $C_{\mathrm{L}}$ are inserted to describe an external force affecting the transducer. In a free oscillating system the load components $C_{\mathrm{L}}$ and $R_{\mathrm{L}}$ are equal to zero. 


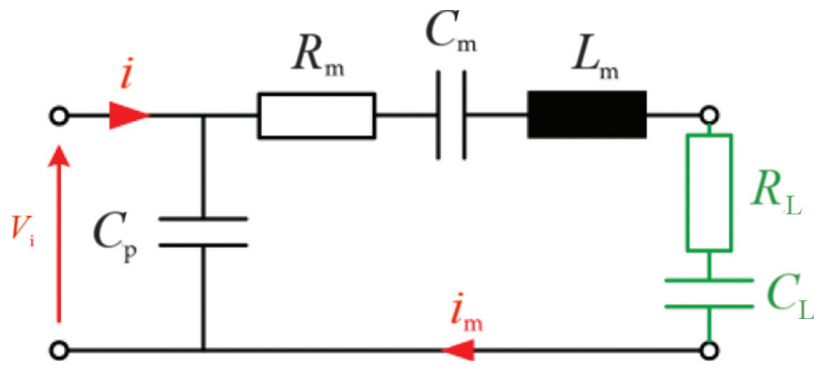

Figure 1: Equivalent electrical model of a piezoelectric transducer.
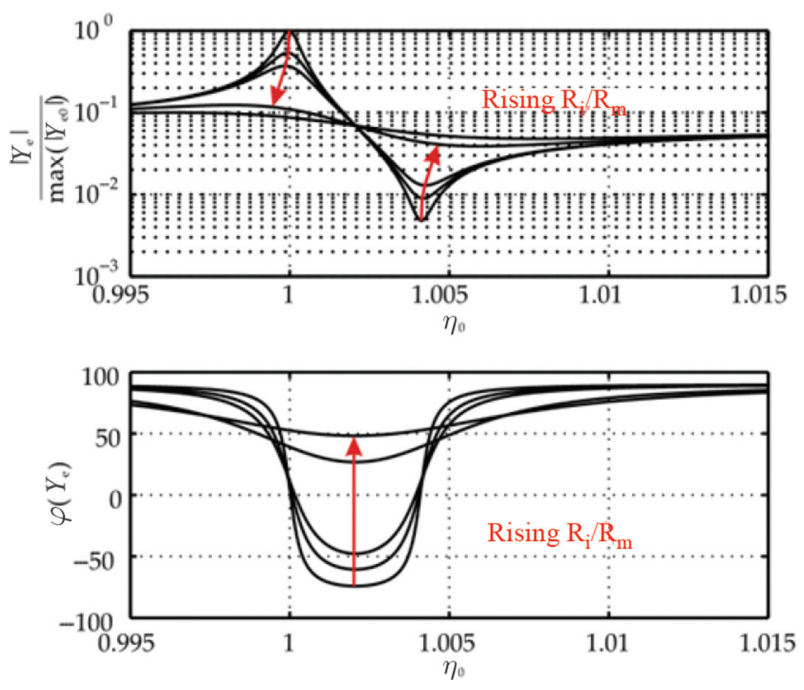

Figure 2: Frequency responses of a piezoelectric transducer with resistive loads.

On the contrary a transducer influenced by a process has in general time-variant load behavior. Thus the load resistance and capacitance can change in time with a high dynamic. The behavior in frequency domain for steady state conditions can be derived from Figure 2. The resonance peak of a piezoelectric transducer decreases with a growing value of the resistive load $R_{\mathrm{L}}$. A load capacitance has an influence on the resonance frequency of the system. A rising load capacitance leads to a frequency shift to a higher resonance and vice versa. To keep driving the transducer in its resonance the implemented resonance feedback control has to be faster than the load dynamic. The curves in Figure 2 are normalized to the eigenfrequency and the maximum of the admittance curve of the unloaded system.

The circuit shown in Figure 1 is characterized by eq. [1]. For a free oscillating system the terms consisting $R_{\mathrm{L}}$ and $C_{\mathrm{L}}$ disappear. Typical equivalent electrical parameters are listed in Table 1 as introduced by Littmann, Hemsel, and Wallaschek (2004):

$$
Y_{\mathrm{e}}=\frac{1}{j \omega L_{\mathrm{m}}+R_{\mathrm{m}}+\frac{1}{j \omega C_{\mathrm{m}}}+\frac{1}{j \omega C_{\mathrm{L}}}+R_{\mathrm{L}}}+j \omega C_{\mathrm{p}}
$$

Table 1: Typical equivalent electrical parameters of an ultrasonic transducer (Littmann, Hemsel, and Wallaschek 2004).

\begin{tabular}{ll}
\hline Parameter & Value \\
\hline$R_{\mathrm{m}}$ & $13.9 \Omega$ \\
$L_{\mathrm{m}}$ & $119 \mathrm{mH}$ \\
$C_{\mathrm{m}}$ & $530 \mathrm{pF}$ \\
$C_{\mathrm{p}}$ & $17.5 \mathrm{nF}$ \\
\hline
\end{tabular}

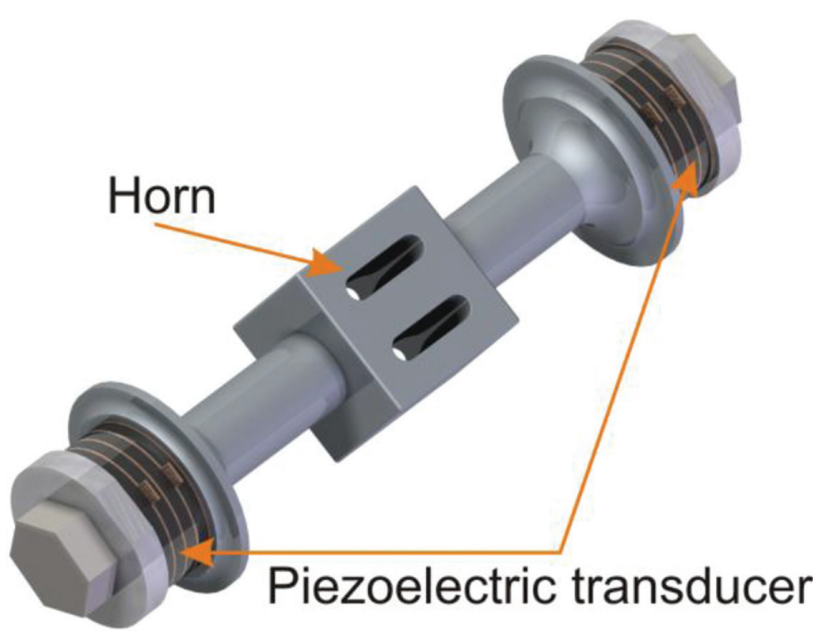

Figure 3: High power piezoelectric transformer.

a)

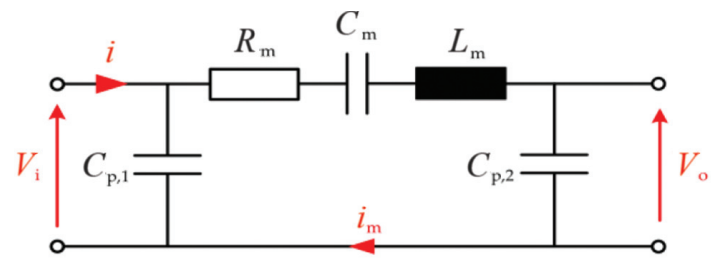

b)

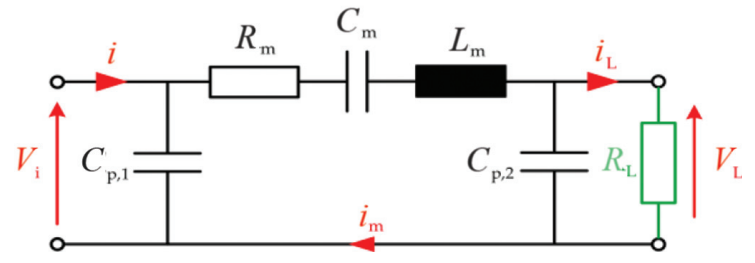

Figure 4: Equivalent electrical model of a piezoelectric transformer (a) open terminals (b) with load resistance.

\section{Piezoelectric Transformer}

Figure 3 shows the structure of the used piezoelectric transformer. The transformation done by the horn between the both piezoelectric transducers is $1: 1$, but can be freely selected to achieve a desired behavior. The equivalent electrical circuit is shown in Figure 4. The transducers 
are designed for longitudinal vibration as same as the horn where the first longitudinal mode of the both transducers is equal to the first longitudinal mode of the horn. The whole system can be considered as a continuum with three nodes and free oscillating ends.

It consists of two parallel capacitances and a series resonant circuit composed of a resistance $R_{\mathrm{m}}$, a capacitance $C_{\mathrm{m}}$ and an inductance $L_{\mathrm{m}}$. The input voltage $V_{\mathrm{i}}$ drives the transformer in resonance. In regard to the open terminals the load is the secondary capacitance $C_{\mathrm{p}, 2}$. In case of shortcircuited terminals, the resistance $R_{\mathrm{L}}$ is equal to zero, the current $i_{\mathrm{m}}$ is equal to the current $i_{\mathrm{L}}$. Thus the piezoelectric transformer behaves like an unloaded transducer in case of short-circuited terminals. By increasing the load on the secondary side the system becomes more damped till a certain value. The system damping decreases at further increase of the resistive load due to the secondary parallel capacitance. A resonance frequency shift occurs through the influence of the secondary parallel capacitance.

The equivalent electrical circuit of a piezoelectric transformer shown in Figure 4 is described by eq. [2] This equation includes the load behavior of a passive resistive load:

$$
Y_{\mathrm{e}}=\frac{1}{j \omega L_{\mathrm{m}}+R_{\mathrm{m}}+\frac{1}{j \omega C_{\mathrm{m}}}+\frac{R_{\mathrm{L}}}{j \omega C_{\mathrm{p}} R_{\mathrm{L}}+1}}+j \omega C_{\mathrm{p}}
$$

In eq. [1] the secondary parallel capacitance $C_{\mathrm{p}, 2}$ is assumed to be equal to the capacitance $C_{\mathrm{p}, 1}$ belonging to the driven ceramics of the transformer. A piezoelectric transformer is explained in detail by Littmann et al.

\section{Behavior of a Piezoelectric Transformer with Passive and Active Load}

Frequency responses with varied passive load are shown in Figure 5 to demonstrate the influence of a rising resistive load. The plotted curves are calculated using eq. [2] and are normalized to the eigenfrequency and the maximum admittance of the unloaded system.

The parameters used for Figure 5 are derived from a frequency response measurement of a piezoelectric transformer with an eigenfrequency near to $20 \mathrm{kHz}$. From Figure 5 one can see the influence of the resistive load and the secondary parallel capacitance. If $R_{\mathrm{L}}$ is equal to zero, the terminals on the right side are short circuited and there is no influence by the capacitive load $C_{\mathrm{p}, 2}$. Increasing of the resistance value leads to a higher system damping and a resonance frequency shift in direction of the antiresonance. Thus the influence of the parallel
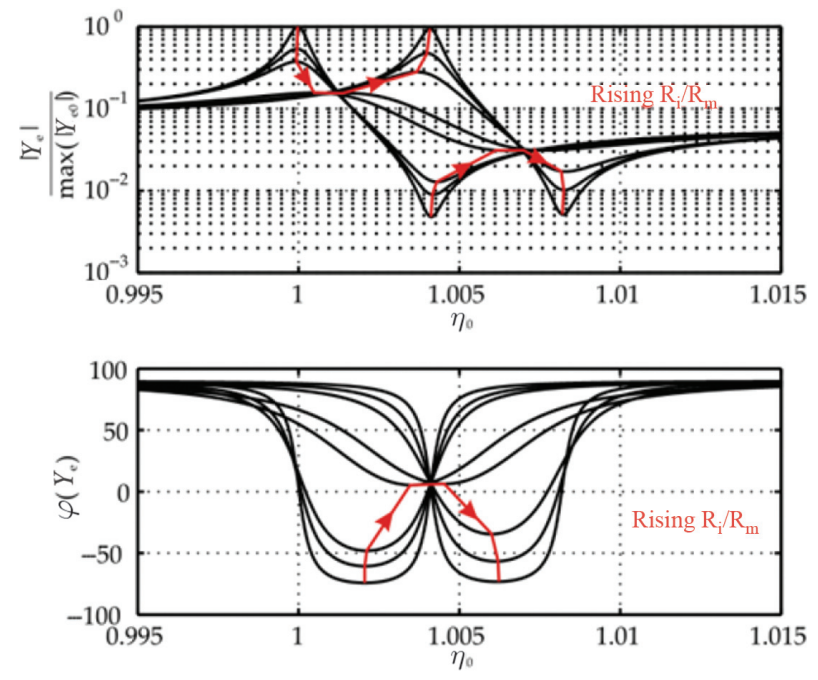

Figure 5: Frequency responses of a piezoelectric transformer with resistive loads.

capacitance $C_{\mathrm{p}, 2}$ rises with rising resistive load $R_{\mathrm{L}}$. In case of open terminals the load is solely described by the parallel capacitance, which is connected in series to the capacitance $C_{\mathrm{m}}$ of the oscillating circuit. Hence the resonance frequency is shifted in antiresonance of the short circuited system. In this case there is no resistive load and the magnitude of the system admittance is equal to an unloaded piezoelectric transducer. However, the passive load has to be changed with a high dynamic to realize the emulation of a certain process. Using passive resistances one can switch between several loads, which leads to undesired switching behavior during switching event. Due to the fact that piezoelectric ceramics exhibit capacitive characteristics current peaks occur and disturb the load emulation. Thus another way has to be found to realize continuous, high dynamic process emulation for high-power systems. This can be done by replacement of the passive through an active load.

Figure 6 shows the simplified principle of the active load emulation using the Ohm's law. A voltage source replaces the passive resistance in Figure 4(b). The load

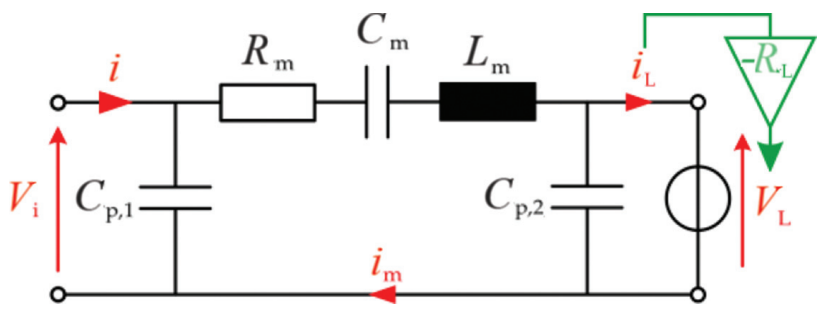

Figure 6: Principle of the active damping using a piezoelectric transformer. 
current $i_{\mathrm{L}}$ can be measured to regulate the voltage $V_{\mathrm{L}}$ to be proportional to the current. This approach produces a continuous, high dynamic load with no undesired switching characteristics using low-cost components.

\section{Hardware and Experimental Setup}

The experimental setup includes two audio amplifiers in parallel connection which deliver a maximum power of $4,000 \mathrm{~W}$ each and produce a voltage up to $200 \mathrm{~V}$. These low-cost audio amplifiers have a relatively high bandwidth and are suitable for the application described in this paper. There is one disadvantage. Many ultrasonic applications need voltages in the range of several kilovolts. For these applications a modification has to be done shown in Figure 7. To achieve higher voltages an electric transformer is inserted between the load emulating amplifier and the piezoelectric transformer. To minimize the desired voltage for different load curves, it is also possible to realize the transformation using a horn which creates an amplitude transformation like a usual piezoelectric transformer.

In this case, there is no need of an electrical transformer. The disadvantage is the higher complexity of the horn.

Functional block diagram of the load emulation system is shown in Figure 8. The primary side of the piezoelectric transformer is driven by a phase locked loop system with integrated phase and current feedback control which is developed by the Institute of Dynamics and Vibration Research. The secondary side of the piezoelectric transformer is connected to the secondary power amplifying stage which consists of two amplifiers of the same style like the driving amplifier. An electrical transformer steps up the amplifier output voltage to the desired stage. The implemented filter generates a load current proportional voltage. A dSpace system is used in the setup to control the magnitude of the filter output voltage. A functional

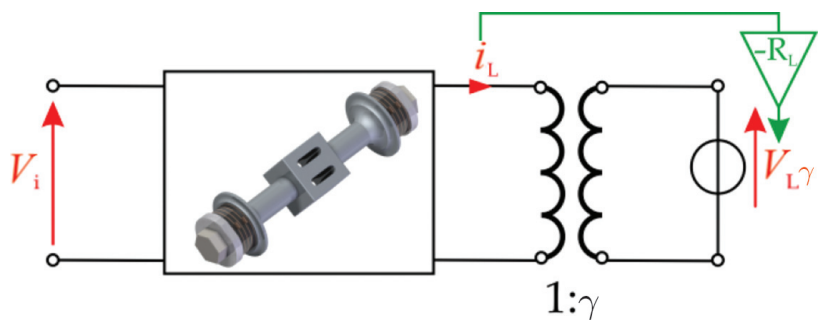

Figure 7: Modified load emulation system with an electric transformer.

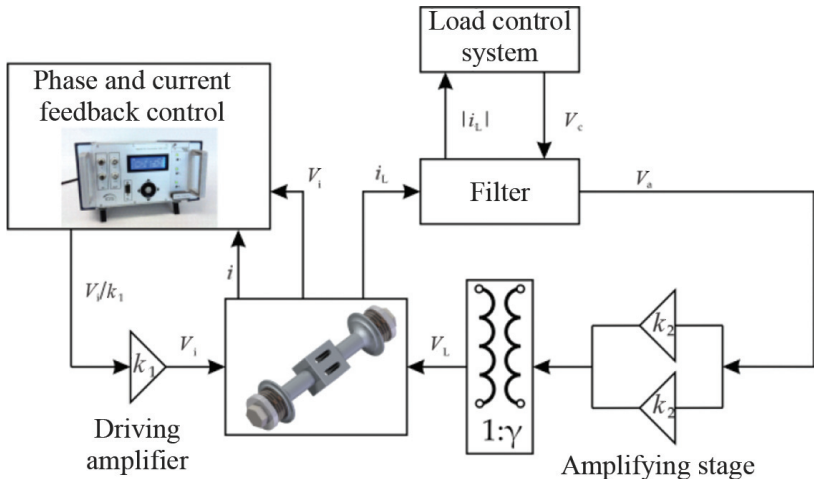

Figure 8: Functional block diagram of used experimental setup.

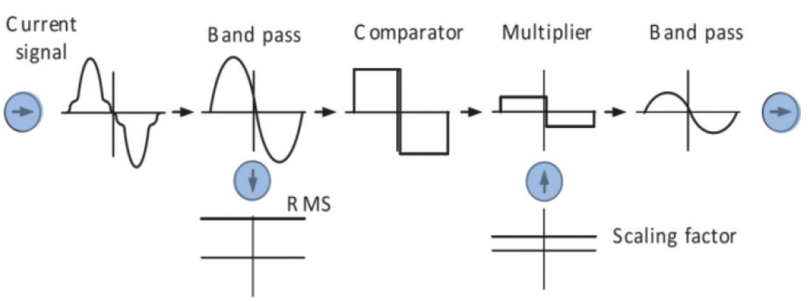

Figure 9: Functional overview of the filter system (Mojrzisch and Twiefel, 2013).

overview of the filter system can be derived from Figure 9. To get the velocity proportional driving current signal of the fundamental harmonic a bandpass filter is used. This prevents a damping of an overtone instead of the desired frequency. The comparator generates an output signal with the desired phase shift of $180^{\circ}$. The output is filtered again to get a sine wave characteristic at the input of the amplifying stage. Using an root mean square (RMS) detector the output magnitude is controlled through a control voltage which is proportional to the output.

\section{Experimental Results}

A frequency response measurement is shown in Figure 10. The system behavior is the same as expected and shown in Figure 5. The measurements were generated using constant emulated loads. The magnitude of the admittance decreases with a rising load. The resonance frequency shifts in the direction of the antiresonance due to the influence of the secondary parallel capacitance $C_{\mathrm{p}, 2}$. Furthermore, calculated frequency responses for passive loads of the same size like the emulated are shown in Figure 10. The measurements and calculations are in good agreement. 


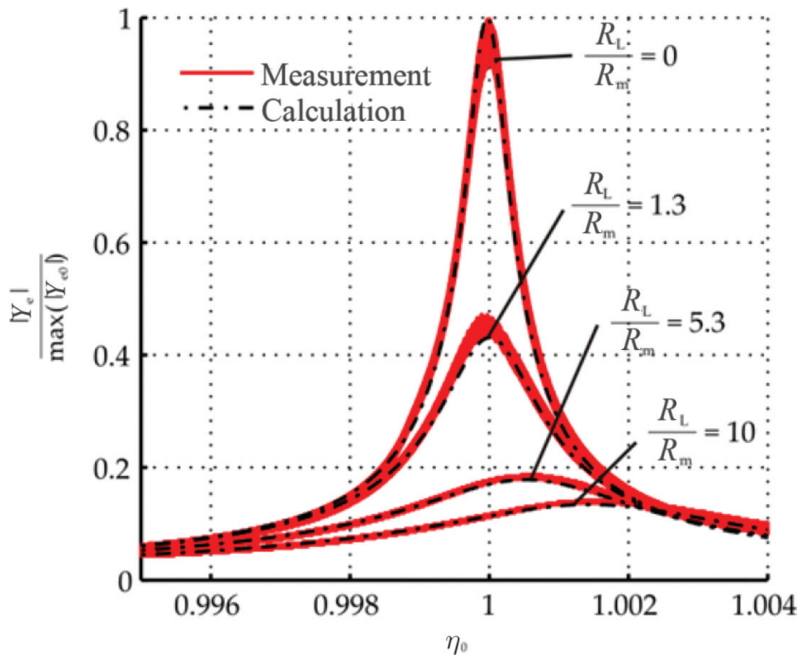

Figure 10: Admittance of the driven transducer with emulated resistive loads.

As Figure 10 shows, the process emulation system can generate constant resistive loads. However, the aim of the presented system is to emulate high dynamic, continuous processes.

Figure 11 shows such a process. The curve of the resistive load is stored inside the load control system and changes with a frequency of several kilohertz. The driving current on the primary side of the transformer decreases with rising load. The inverse load behavior one can see within the driving current. Figure 11 shows the desired load emulation behavior.

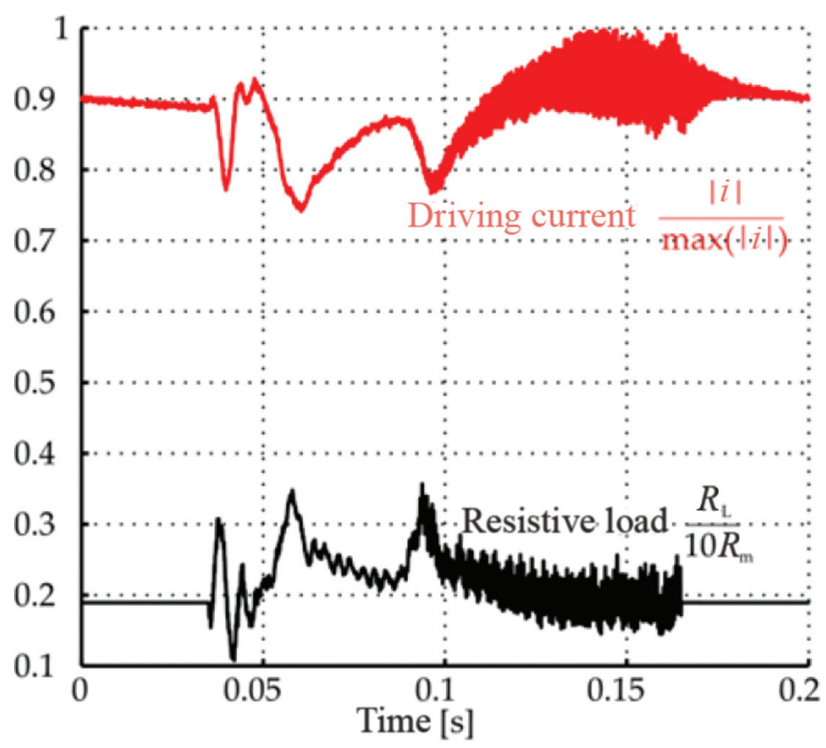

Figure 11: Driving current behavior using a resistive load curve.
Here, the driving current is normalized to its maximum and the ratio between the emulated and internal resistance is used to show the high dynamics of the stored load curve.

\section{Conclusion}

A high dynamic, flexible load emulation system is presented by this paper. The present system allows tests of a wide field of feedback control algorithms. This decreases design periods of feedback-controlled driving systems for ultrasonic actuators. Long-term tests with a high number of cycles by utilization of low-cost components are possible. The high-power load emulation system solely consists of low-cost audio amplifiers and load controlling electronics. The load can be continuously changed by the presented system without undesired switching characteristics with a frequency up to $10 \mathrm{kHz}$. The design is done for high-power applications up to $2 \mathrm{~kW}$ and a voltage stage of up to $2 \mathrm{kV}$, but it also can be used for low-power applications.

\section{References}

Ahmed, N., A. V. Mitrofanov, V. I. Babitsky, and V. V. Silberschmidt. 2007. "Analysis of Forces in Ultrasonically Assisted Turning." Journal of Sound and Vibration 308, 845-854, 2007.

Babitsky, V. 2004. “Autoresonant Control of Ultrasonically Assisted Cutting." Mechatronics 14, 91-114, 2004.

Hemsel, T., and S. Priya. 2006. "Model Based Analysis of Piezoelectric Transformers.” Ultrasonics 44, 741-745, 2006.

Kauczor, C., and N. Fröhlecke. 2004. Inverter Topologies for Ultrasonic Piezoelectric Transducer with High Q-Faktor. $35^{\text {th }}$ Annual IEEE Power Electronics Specialists Conference.

Littmann, W. 2001. Piezoelektrische, resonant betriebene Ultraschall-Leistungswandler mit nichtlinearen mechanischen Randbedingungen. Ph.D. Thesis, University of Paderborn. (in German)

Littmann, W., T. Hemsel, and J. Wallaschek. 2004. Design criteria for piezoelectric transformers, The $18^{\text {th }}$ International Congress on Acoustics

Maas, J., T. Schulte, and N. Fröhleke. 2000. "Model-Based Control for Ultrasonic Motors." ASME Transactions on Mechatronics 5, 165-180, 2000.

Mojrzisch, S., and J. Twiefel. 2013. On ultrasonic squeeze film levitation: modeling and feedback control of ultrasonic bearing systems. SPIE Smart Structures and Materials + Nondestructive Evaluation and Health Monitoring, San Diego, USA, 2013.

Twiefel, J., M. Klubal, C. Paiz, S. Mojrzisch, and H. Krüger. 2008. Digital signal processing for an adaptive phase-locked loop controller. Proc. SPIE 6926, Modeling, Signal Processing and Control for Smart Structures, San Diego, USA, 2008. 